\title{
Study of Magnetic Interference on a LVDT Prototype
}

\author{
Alessandro Masi ${ }^{1}$, Alessandro Danisi ${ }^{1,2}$, Roberto Losito $^{1}$, Michele Martino ${ }^{1,3}$, Giovanni Spiezia ${ }^{1}$.
}

\author{
${ }^{1}$ CERN, European Organization for Nuclear Research \\ CH-1211 Geneva 23, Switzerland \\ ${ }^{2}$ École Polytechnique Fédérale de Lausanne (IMT-LAI) \\ CH-1015 Lausanne, Switzerland \\ ${ }^{3}$ University of Naples "Federico II",
}

\begin{abstract}
This paper presents a prototype of Linear Variable Differential Transformer, which has been designed, simulated and produced in order to observe, study and characterize the effects on its working of an external interfering magnetic field. The LVDT prototype has been first simulated, both in standard conditions and in case of external interference, by using the FEM software FLUX. The design guidelines coming from the simulations have been used to develop the prototype and a complete set of measurements has been performed on it. A comparison between simulations and measurements is then proposed.
\end{abstract} Method.

Keywords- LVDT, Magnetic Interference, Finite Element

\section{INTRODUCTION}

The Linear Variable Differential Transformer is a magnetic position transducer. Its use is very spread due to its features of being a contactless sensor, with infinite resolution and a high accuracy [1]. The latter can be also improved by using proper conditioning electronics [2]-[4] and the ratiometric reading technique [5]. Such properties make its use be very common in harsh environments, as nuclear plants and particle accelerators [6].

The LVDT sensor is basically a transformer with one primary winding, in the center of the cylindrical structure, and two secondary windings, one of each side of the primary, wound on a cylindrical support (fig. 1). A ferromagnetic core can move along the axis and the flux linkage between the primary and secondary windings depends accordingly to its position. In particular, when the core is all on one side of the structure, one secondary voltage is maximum, whereas the other one is minimum. When the core is in the middle, the two voltages are equal. The position of the core can be extracted by a differential reading of the secondary signals. The frequency used for the feeding signal is often in the range of the $\mathrm{kHz}$.

Although the LVDT reading accuracy can be guaranteed even in critical and noisy installations [6], this sensor has shown to be sensitive to external slowly varying magnetic fields [7]. This is the case, for example, of installations next to devices with significant leakage or generated fields, such as motors or current cables. The influence of such devices can bring to a position reading error which can be of the order of magnitude of some hundreds of micrometers. This kind of error can represent a serious issue in application where a high
Electrical Engineering Department. 80, P.le Tecchio, 80145 Naples, ITALY. \{Alessandro.Danisi, Roberto.Losito, Michele.Martino, Alessandro.Masi, Giovanni.Spiezia\}@cern.ch

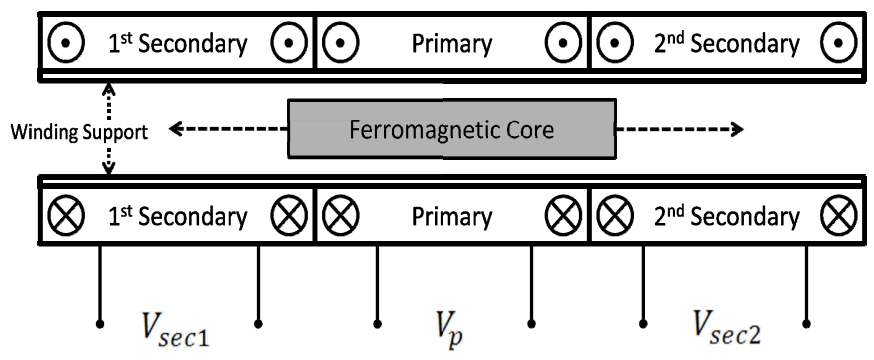

Figure 1. Typical LVDT layout and working principle (longitudinal section).

accuracy is required [6]. This question is taken into account in some LVDT data sheets, but there is no advice regarding the quantitative effect on the position reading, nor the possible countermeasures.

In this paper, a LVDT prototype is presented. Being a custom sensor, its structure and the type of materials are well known. Such a prototype will be used to study and understand the effects of the magnetic interference on its working. This will be the baseline for a future design of a LVDT which can reject the effect of external magnetic fields.

A complete FEM model is built, using the simulator FLUX, for a custom LVDT geometry, and the effect of a DC magnetic field on the measured position is observed. The use of FEM tools for analysis and synthesis processes of LVDT sensors is not new to the scientific literature. As a matter of fact, FEMaided analysis can allow a deeper investigation regarding local variables and physical magnitudes (i.e. local values of magnetic flux density or magnetic field) [8]-[9]. Taking the simulation results as design guidelines, the LVDT prototype has been manufactured and a complete set of measurements has been performed, in order to investigate the working in standard and in interference conditions.

In section II, after the presentation of the finite element model of the sensor, the LVDT prototype is presented and the complete simulation plan is discussed. In section III, the measurements setup and the results are shown and a comparison with the simulations is done.

\section{PRototype Design AND SIMULATION}

\section{A. LVDT Finite Element Model}

The FEM model for the simulation of the LVDT sensor has been developed using the simulation software FLUX. This 


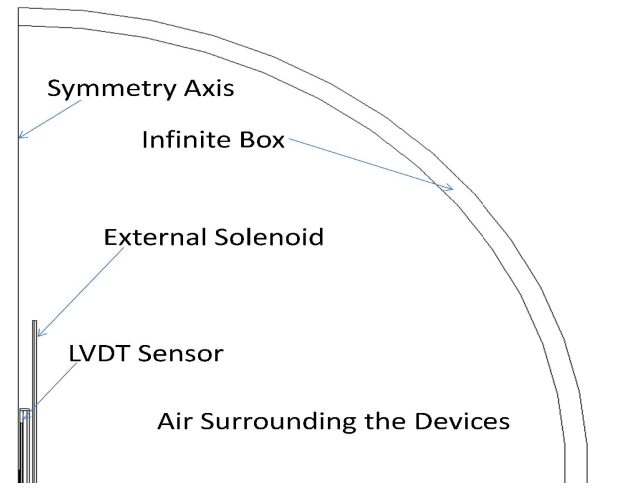

Figure 2. Half of the 2D Longitudinal section of the simulated LVDT geometry and external solenoid. In the infinite box a geometrical transformation is performed in order to simulate the infinite space [10].

simulator is particularly suited for the finite element analysis of electromagnetic problems involving 2D and 3D geometries [10].

The simulated model slightly differs from the simple structure presented in section I and displayed in fig. 1. Actually, in this new model the primary coil is wound on the entire length of the winding support, whereas the secondary coils are wound over the primary, one of each side of the structure. In this way, the leakage inductances of the transformer are significantly reduced. The model presents insulator washers and layers, treated as nonmagnetic regions.

The structure is enclosed in a ferromagnetic cylindrical case with two end caps, made of ferromagnetic steel, as for the winding support, a material which exhibits relatively high magnetic permeability. The core is a hollow cylinder, whose length is equal to the secondary coils length, made of a $48 \%$ $\mathrm{Ni}-\mathrm{Fe}$ alloy. This magnetically soft material exhibits very high magnetic permeability and is commonly used for these applications [11]-[12].

Since the structure has complete cylindrical symmetry, the simulation geometry has been built in the $2 \mathrm{D}$ environment and only half of the longitudinal section of the geometry has been simulated, as shown in fig. 2. In this figure, half of the simulated geometry is displayed. In fact, due to the multiple symmetries of the sensor, the other half (which should be in the bottom) is identical. However, it has been taken into account in the simulations, because this second symmetry is only valid for the LVDT geometry when the core is in the center, since the core can break the symmetry with its movement.

The structure is essentially a high aspect ratio geometry. For this reason, a fine mesh has been chosen in order to quantize the thicknesses, whereas the mesh density along the length of the sensor can be smaller. The meshing parameters for the geometry are represented in table I.

A remark has to be pointed out regarding the simulation of materials and their properties. No heat treatment (i.e. annealing procedure) has been applied on the materials of the prototype developed for the magnetic interference study. In these conditions, the materials properties are different with respect to the annealed case, in which both the Ni-Fe alloy and the ferromagnetic steel exhibit high permeability and low hysteresis losses. Therefore, the materials have been described in the simulations with a permeability which is lower than the value which one can observe when the materials are in annealed conditions, in order to take into account the degradation of relative magnetic permeability due to the absence of annealing procedure. In addition, the materials exhibit a reduced value of resistivity in simulation, in order to include the hysteresis losses (that are relevant in not annealed state) in the losses computed by the simulator.

TABLE I. MESH INFORMATION

\begin{tabular}{|l|c|}
\hline \multicolumn{1}{|c|}{ Mesh Info } & Mesh Parameters \\
\hline Mesh Type & 2D Triangular Mesh \\
\hline $\begin{array}{l}\text { Mesh Order and } \\
\text { Density }\end{array}$ & 2nd Order, Non-uniform \\
\hline $\begin{array}{l}\text { General Meshing } \\
\text { Number of Poor } \\
\text { Elements }\end{array}$ & 2-3 elements per thickness \\
\hline Number of Nodes & About $4 \%$ \\
\hline
\end{tabular}

\section{B. Simulation Results}

The working of the LVDT FEM model has been analyzed in simulations. In particular, different simulations have been done in order to work out the LVDT characteristic curve, which shows the secondary voltages with respect to the core position. In this framework, the sensor has been supplied by a $3.5 \mathrm{~V}$ peak sinusoidal voltage at $2000 \mathrm{~Hz}$. We computed the first harmonics of the signals. The result is displayed in fig. 3 (continuous line) in the range of core positions going from -20 $\mathrm{mm}$ to $20 \mathrm{~mm}$.

We can notice a dual behavior of the curve in terms of monotonicity, as well as a non-linearity of the relationship. The linearity becomes more acceptable when we reduce the core position range around the null position (for example, from -10 $\mathrm{mm}$ to $10 \mathrm{~mm}$ ). The curve is symmetric with respect to the null position, due to the complete symmetry of the device. The simulations show that the secondary transformation ratio of the sensor goes from 0.38 (when the core is not coupled) to 0.64 (full core coupling).

The behavior of the ratiometric with respect to the position has been investigated too and the relative results are depicted in figure 4 (continuous line). The ratiometric is a monotonic function rising from about -0.25 to about 0.25 . Once again, the perfect odd symmetry of the curve reflects the perfect geometrical symmetry and reciprocity of the simulated device.

The presence of an external magnetic field impinging the LVDT structure has also been considered in the simulations. Since the LVDT is more sensitive to a longitudinal field, rather than to a transversal one [13], the case of a longitudinal interfering DC field has been simulated.

The DC interfering field has been generated in the simulations by means of an external solenoid, coaxial with the sensor (fig. 2). The LVDT is inside the solenoid, whose length assures a uniform field distribution along the axis. 


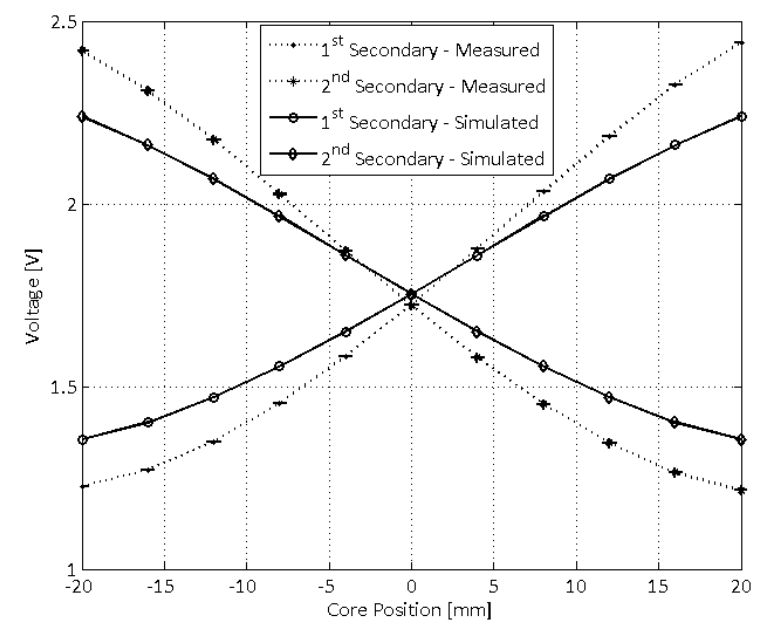

Figure 3. Simulations and Measurements results regarding the LVDT secondary characteristic curve. The continuous lines refer to simutations, whereas the dotted lines refer to measurements results. The measurement data are depicted with the corresponding uncertainty.

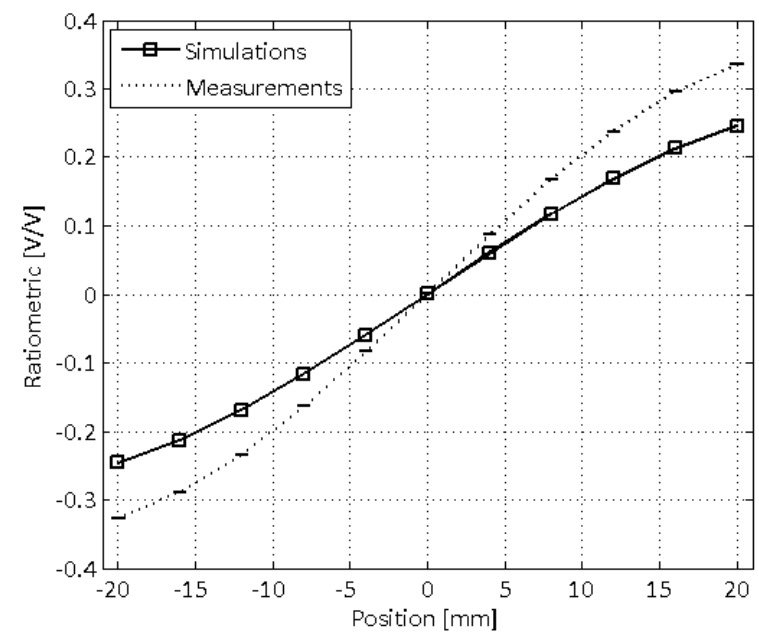

Figure 4. Simulations and Measurements results regarding the ratiometric. The measurement data are depicted with the corresponding uncertainty.

In these conditions, a set of simulation has been planned and performed. The case of a $1 \mathrm{mT}$ external magnetic flux density has been here considered, which in air corresponds to an interfering magnetic field of about $800 \mathrm{~A} / \mathrm{m}$. The core is moved inside the sensor from $-20 \mathrm{~mm}$ to $20 \mathrm{~mm}$. The position reading has been performed using the ratiometric reading technique and through a proper calibration curve, which has been built in case of no external interference.

At each core position, a position drift has been computed as the difference between the position in presence of the external interference and the position in absence of the interference (which, in simulation, is exactly the real position of the core).

The results are displayed in fig. 5. Some interesting remarks can be pointed out by looking at this graph. The position drift is

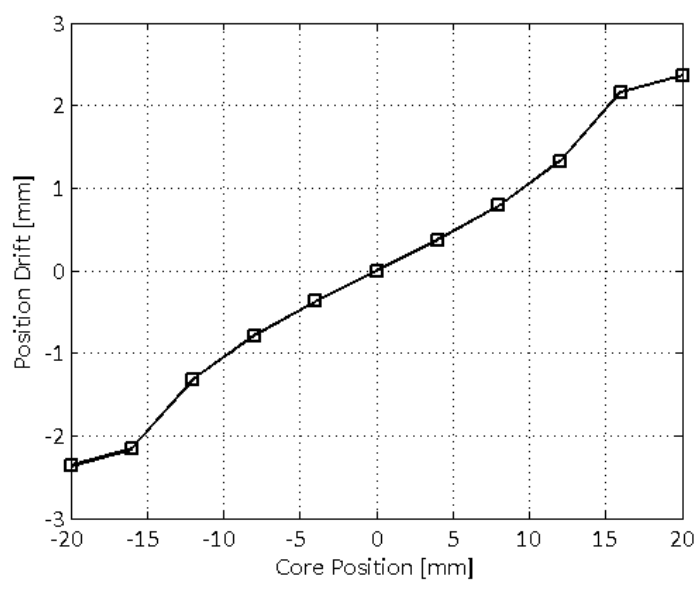

Figure 5. Position drift in simulation, in case of $1 \mathrm{mT}$ longitudinal interfering flux density.

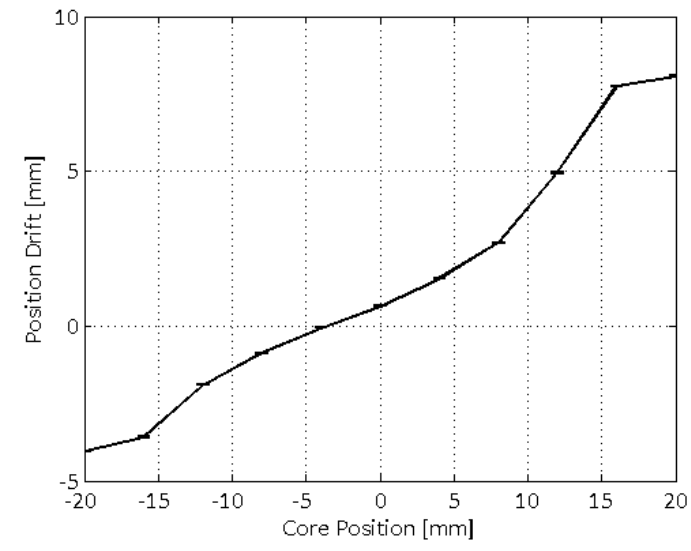

Figure 6. Position drift in measurement, in case of $1 \mathrm{mT}$ longitudinal interfering flux density. The data are depicted with the corresponding uncertainty.

an odd monotonic function of the core position and in null position it is null on turn. The farther from the center the core is, the worse the position reading is. In the worst case, in which the core position is $20 \mathrm{~mm}$, the position drift can reach a value of about 2.3 millimeters.

\section{Prototype Realization}

The simulation work which has been just presented constitutes the starting point for a proper LVDT prototype manufacturing. As a matter of fact, the simulation results suggest important design guidelines regarding: i) the sensor geometry, in order to minimize the leakages, ii) the magnetic materials properties, in order to have good linearity and sensitivity.

Looking at these guidelines, a LVDT prototype has been manufactured. The sensor dimensions and geometry reflect the simulation criteria, as well as the choice of the materials that compose the magnetic path of the sensor (core, winding support, external case and end caps). As already said in the last section, the annealing process is not performed on the materials. 
A number of turns of 1600 has been chosen for the primary coil, in order to have a sufficiently high field inside the sensor, whereas a number of turns of 850 has been chosen for both secondaries, setting the transformation ratio. The primary coil has been wound on 4 layers with a wire diameter of $0.28 \mathrm{~mm}$ (in order to allow possible measurements even with high currents), whereas the secondaries are single layer coils with a wire diameter of $0.06 \mathrm{~mm}$.

\section{MEASUREMENT Results}

A complete automatic test bench, whose details can be found in [14], has been used for the LVDT prototype metrological characterization, in both standard and magnetic interference conditions.

As for the simulations, the external longitudinal magnetic field is generated by means of an external calibrated solenoid, fed by a DC current, which encloses the LVDT sensor, so to have the desired amplitude and a uniform distribution for the field along the solenoid length. In addition, a demagnetization process is performed and verified for each measurement step, in order to demagnetize the magnetic circuit of the sensor and perform each measurement in the same static working point of the equivalent B-H curve.

The LVDT characteristic curve has been measured in the core positions ranging from $-20 \mathrm{~mm}$ to $20 \mathrm{~mm}$. The result is depicted in fig. 3 (dotted line). In the graph, on each point, the expanded measurement uncertainty is shown. This has been evaluated via the standard deviation of 30 repeated measurements with coverage factor 3 [14].

As in the FEM analysis, a non linearity of the relationship has been observed, especially for high core position ranges. The measurements showed that the secondary transformation ratio of the sensor goes from 0.36 (against a value of 0.38 for the simulations) to 0.69 (against a value of 0.68 for the simulations).

Regarding the voltage amplitudes, the agreement between simulation results and measurements is good, since the voltage first harmonics of the characteristic curve are different in the worst case of about $15 \%$ in the nominal core position range, whereas this difference decreases to $6 \%$ when reducing to [-10 $\mathrm{mm}, 10 \mathrm{~mm}$ ] the core position range.

As for the simulations, the behavior of the ratiometric has been analyzed in the measurements too. The results are depicted in figure 4 (dotted line). In this case, the ratiometric is a monotonic function of the position rising from about -0.32 (against a value of -0.25 for the simulations) to about 0.34 (against a value of 0.25 for the simulations). In addition, the curve exhibits an evident nonlinearity. The graph in figure 4 allows noticing the influence of the ratiometric reading on the match between simulations and measurements. As a matter of fact, while the match regarding the secondary voltages is of about $16 \%$ in the worst case, the one regarding the ratiometric is around $35 \%$. Furthermore, such effect does not act uniformly on all the positions.

The measurements in presence of a $1 \mathrm{mT}$ external longitudinal magnetic flux density have been performed as well on this prototype and the related position drift is presented in fig. 6. The uncertainty on this measurement values has been calculated applying the uncertainty propagation law on the uncertainty on the ratiometric at each core position, which is obtained by repeated measurements [14].

Some differences with respect to simulations have to be explained and are source of accurate study. First of all, the drift at $0 \mathrm{~mm}$ is zero in the simulations and non null in the measurements. As a matter of fact, the simulated structure has been built having as hypothesis the perfect cylindrical symmetry of the device and the complete symmetry around the center of the sensor, when the core is in null position. These properties bring to equal effects of the external magnetic field on the secondary voltages, when the core is in null position. Due to the ratiometric reading, the position drift in this case is zero. In the case of the prototype, the position drift at $0 \mathrm{~mm}$ is not zero because the perfect symmetries are not assured (imperfect geometry shapes, nonhomogeneous magnetic properties due to the absence of annealing process, coiling imperfections). This statement can be confirmed even by noticing thatthe drift has different values in modulus in the extreme positions $(-20 \mathrm{~mm}$ and $20 \mathrm{~mm})$, in the measurement results.

From the point of view of the trend, one can see that the measured drift is an odd monotonic function of the core position and actually the trend matches the one coming from the simulations.

The differences of the drift values may be due to two main causes. Firstly, the absence of annealing treatment on the materials makes the magnetic properties be non homogeneously distributed on the magnetic media and may cause incongruence with the simulated model. In addition, the ratiometric reading technique magnifies (by definition) the disagreement between simulations and measurements, not even uniformly, as already seen. Therefore, the agreement regarding the position drift is an open issue and needs further simulations and measurements.

\section{CONCLUSIONS AND OUTLOOK}

In the complete framework of the LVDT magnetic interference study, the LVDT finite element analysis is used in this work to address the design of a custom LVDT prototype. A complete FEM model of the sensor has been presented, together with simulation results of the LVDT characteristic curve and the effect of a $1 \mathrm{mT}$ external flux density on the position reading. On this basis, a custom LVDT prototype has been manufactured.

A measurement test bench [14] has been used to characterize the prototype and verify the simulation results. The agreement with the simulations has been discussed in all cases. In particular, good agreement has been observed for the LVDT secondary characteristic curve (percentage difference of $16 \%$ in the nominal core position range), whereas deeper investigations are needed for the agreement regarding the ratiometric and the position drift.

Although some considerations have been proposed for the behavior of the position drift with the core position, the study of a complete analytical model of the magnetic interference effect on the LVDT sensor, when this is supplied in voltage, 
outgoes the scope of this work. Anyway, an analytical model for the magnetic interference on LVDTs, when the supply is performed with a current signal, can be found in [15]. The study of an analytical model in voltage, together with further measurements with different materials and particular adjustments of simulation parameters are currently matter for future works.

Possible incongruence in the magnetic materials features and defined magnetic properties (e.g. with annealing process) are the main key points for the future that this work has shown. Nevertheless, since good agreement has been shown for the secondary characteristic curve and differences have been pointed out regarding the ratiometric and the position drift, the future study would have not only to deal with the investigation on the incongruence of magnetic properties, but also on the way the incongruence propagates itself when elaborating the signals.

\section{ACKNOWLEDGEMENTS}

The authors would like to thank Eng. Antonio Pierno and Dr. Daho Taghezout for the support on measurements and simulations respectively. They would also like to thank Prof. Vittorio Giorgio Vaccaro, Prof. Felice Cennamo and Prof. Yves Perriard for their useful discussions and suggestions.

\section{REFERENCES}

[1] D. S. Nyce, "The LVDT," in Linear Position Sensors, Hoboken, NJ: Wiley-Interscience, 2004, pp. 94-97.

[2] A. Masi and R. Losito, "LHC Collimators low level control system," IEEE Trans. Nucl. Sci., vol. 55, no. 1, pp. 333-340, Feb. 2008.

[3] R. S. Weissbach, D. R. Loker and R. M. Ford, "Test and Comparison of LVDT Signal Conditioner Performance," Proc. 17th IEEE Instrumentation Meas. Technol. Conf., 2000, pp. 1143-1146.
[4] D. Crescini, A. Flammini, D. Marioli and A. Taroni, "Application of an FFT-based Algorithm to Signal Processing of LVDT Position Sensors," IEEE Trans. Instrum. Meas. vol. 47, no. 5, pp. 1119-1123, Oct. 1998.

[5] K. Ara, "A Differential Transformer With Temperature- and ExcitationIndependent Output," IEEE Trans. Instrum. Meas. vol. IM-21, no. 3, pp. 249-255, Aug. 1972.

[6] A. Masi, A. Brielmann, R. Losito, and M. Martino, "LVDT conditioning on the LHC collimators," IEEE Trans. Nucl. Sci., vol. 55, no.1, pp. 6775, Feb. 2008.

[7] M. Martino, A. Danisi, R. Losito, A. Masi, G. Spiezia, "Design of a LVDT with high rejection to external interfering magnetic field," IEEE Trans. Mag., vol. 46, no. 2, pp. 674-677, Feb. 2010.

[8] J. K. Sykulski, E. Sykulska, and S. T. Hughes, "Application of finite element modelling in LVDT design," COMPEL, vol. 11, no. 1, pp. 7376, 1992.

[9] M. Repetto and J. Simkin, "Engineering analysis for design optimization of differential transformers," IEEE Comput.-Aided Eng. J., vol. 5, no. 2, pp. 51-53, Apr. 1988.

[10] Flux ${ }^{\circledR} 9$ 2D/3D Applications User's Guide, CEDRAT, Meylan, FR, 2006.

[11] R. Bozorth, Ferromagnetism, 3rd ed. New York: D. Van Nostrand Company, 1951.

[12] K. Hoselitz, "Magnetically soft materials," in Ferromagnetic properties of Metals and Alloys, Clarendon Press (Oxford), GB: Oxford Univ. Press, 1952, pp. 238-239.

[13] A. Danisi, "Simulation of DC Interfering Magnetic Field Effects on the LHC Collimators' LVDT Positioning Sensors," M.S. thesis, Dept. Electron. Eng., Univ. "Federico II", Naples, IT, 2009. https://edms.cern.ch/, EDMS Id 1016998.

[14] G. Spiezia, R. Losito, M. Martino, A. Masi, A. Pierno, "An Automatic Test Bench for the Measurement of the Magnetic Interference on LVDTs," accepted for I2MTC 2010

[15] M. Martino, G. Golluccio, R. Losito, A. Masi, "An analytical model of the effect of external DC magnetic fields on the AC voltages of an LVDT," accepted for I2MTC 2010. 